\title{
Criminologie
}

\section{L’individu et la collectivité}

\section{Alice Parizeau}

Volume 15, numéro 2, 1982

Criminels et psychiatrie

URI : https://id.erudit.org/iderudit/017164ar

DOI : https://doi.org/10.7202/017164ar

Aller au sommaire du numéro

Éditeur(s)

Les Presses de l'Université de Montréal

ISSN

0316-0041 (imprimé)

1492-1367 (numérique)

Découvrir la revue

Citer ce compte rendu

Parizeau, A. (1982). Compte rendu de [L'individu et la collectivité].

Criminologie, 15(2), 115-122. https://doi.org/10.7202/017164ar d'utilisation que vous pouvez consulter en ligne.

https://apropos.erudit.org/fr/usagers/politique-dutilisation/ 
On ne peut aborder ce Précis pratique de psychiatrie, sans un profond respect pour une approche scientifique qui s'efforce en même temps de maintenir une unité de présentation et une clarté de style. Oeuvre de plusieurs auteurs, effectuée sous la direction du docteur R. Duguay et du docteur H.F. Ellenberger, elle ne peut être résumée, puisqu'il s'agit d'un précis, c'est-à-dire d'un livre qui constitue un outil de travail pour les médecins généralistes comme pour les étudiants.

Dans la préface à l'édition canadienne, le docteur Arthur Amyot souligne que c'est un livre «qui innove sur plusieurs points, dont... parmi les plus particuliers : l'état des travaux de recherche sur les pathologies du nourrisson, la large place accordée aux découvertes récentes concernant la douleur chronique et la façon originale et clinique d'aborder la cure psychanalytique ».

Paul Sivadon, pour sa part, dans la préface destinée à l'édition française insiste sur l'intérêt particulier «d'une synthèse, enrichie par la perspective transculturelle dont les auteurs sont familiers". "La psychiatrie canadienne-française ", écrit-il, «a eu la chance de se trouver au carrefour des courants français et anglo-saxon. Son mérite est d'avoir su intégrer ces courants, à l'occasion de cette "révolution tranquille " qui, dans les années soixante, a inauguré une mutation sans précédent grâce à laquelle une psychiatrie encombrée d'archaismes a pu, en quelques années, se porter au premier plan du progrès. »

\section{Psychiatrie et criminologie}

Il nous est impossible ici de commenter un manuel de psychiatrie de cette envergure et cela n'est pas de notre propos. Nous allons donc nous limiter surtout à aborder certaines conclusions dégagées dans ses textes par H.F. Ellenberger, le plus susceptible d'avoir un lien direct avec la criminologie puisqu'il a enseigné à l'École de criminologie de l'Université de Montréal pendant plusieurs années et que son apport à cet égard a été non seulement considérable, mais encore tout à fait unique.

* Commentaire sur l'ouvrage de : R. Duguay, H.F. Ellenberger et al, Prícis pratique de psychiatrie, Edit. Chenelière et Stanké, Montréal, et Maloine Éditeur. Paris, 1981,693 pages. 
Les éditeurs de l'ouvrage n'ont pas crû utile, et cela est regrettable, de donner à la fin du volume un bref aperçu des travaux publiés par le passé par plusieurs de ses auteurs. Il n'est donc pas inutile de rappeler que H.F. Ellenberger a à son actif des travaux de recherches et des publications dont l'importance ne saurait être résumée en quelques phrases. Il s'agit là non seulement d'un apport scientifique reconnu au Québec, comme sur le plan international, mais aussi d'une œuvre de pédagogue.

Dans le Précis pratique de pychiatrie, on lui doit en premier lieu le chapitre sur l'histoire de la psychiatrie, puis celui relatif au problème des classifications psychiatriques.

Profondément humaniste, le docteur Ellenberger y affronte à sa façon les problèmes de l'heure en écrivant :

Quelques anti-psychiatres... prétendent que la maladie mentale n'existe pas et considèrent les cas cliniques comme produits artificiels de la répression sociale. Le traitement psychiatrique ne serait qu'une forme de punition déguisée et de violence sociale... (p. 11).

Au lieu de condamner ce courant de pensée, l'auteur conclut en lui opposant des évidences scientifiques, tout en reconnaissant cependant au passage la valeur de certaines critiques.

... les progrès énormes de la pharmacothérapie ont abouti à vider certains hôpitaux psychiatriques d'un grand nombre de leurs malades et c'est alors seulement que certains abus auxquels ils avaient donné lieu parurent scandaleux...

Quelle que soit l'explication que l'on puisse donner à la vague récente de l'anti-psychiatrie, celle-ci aura du moins été utile à la psychiatrie en l'obligeant à une révision de ses bases philosophiques et de ses principes fondamentaux.

Respect de cette opinion scientifique oblige; on ne saurait mettre en doute l'avis d'un spécialiste de la trempe de H.F. Ellenberger. Qu'il nous soit permis toutefois d'observer que ce sont les découvertes dans le domaine de la pharmacothérapie qui permettent désormais de libérer certains malades mentaux violents, sans provoquer la peur de victimisation dans l'opinion publique. Par le passé, ce genre de traitement en dehors des murs des hôpitaux-prisons n'aurait pas été accepté par les collectivités plus soucieuses, par définition, de leur propre sécurité que de certaines conceptions philosophiques pronées par les porte-paroles de l'anti-psychiatrie enfermés dans la tour d'ivoire des théories abstraites.

Une constatation similaire s'applique à l'opinion d'Heinrich Neumann, citée par H.F. Ellenberger (p. 13), selon laquelle : "Il 
n'y a pas de maladies mentales, il n'y a que des malades. " On voit là à quel point les problèmes philosophiques préoccupent les psychiatres d'hier et d'aujourd'hui. Personne, en effet, n'oserait écrire qu'il n'y a pas de lépreux, mais uniquement des malades, sans ajouter que pendant des siècles ils étaient à ce point contagieux qu'on les isolait sur les îles désertes parce que la médecine ne savait pas comment les soigner.

Un malade, objet de commisération et de pitié, cesse de l'être, en pratique quand il menace de façon évidente les autres, qui évitent dès lors par tous les moyens, aussi cruels puissent-ils être, le risque de contact avec lui. Certes ce n'est pas là une approche humaniste, mais plutôt une sorte de reflet des réalités contre lesquelles on ne lutte pas à l'aide des philosophes, mais des découvertes dans le domaine de la science médicale.

À cet égard, mentionnons que le docteur P. Lefebvre, dont on trouvera en page 121 un texte sur les névroses selon la conception freudienne, vient de trouver un médicament permettant de soigner certains types de dépressions et que sa découverte a été publicisée récemment auprès du public. Il est regrettable que l'éditeur québécois, tout du moins, n'a pas jugé utile, ne serait-ce qu'en renvoi en bas des pages, de mentionner les recherches, les publications et les découvertes faites par les auteurs de ce livre. L'absence de professionnalisme est franchement agaçante à cet égard puisqu'elle prive les lecteurs des points de repères fort intéressants.

Mais revenons à l'analyse succincte des textes signés par H.F. Ellenberger et particulièrement à ceux qui, comme nous l'avons mentionné plus haut, sont les plus liés à notre discipline. Dans deux chapitres, dont celui sur les schizophrénies et sur les délires chroniques, l'auteur retient expressément les aspects criminologiques (p. 295,333 ). Une fois de plus il fait preuve là d'un humanisme et d'une immense culture.

" Pendant longtemps, écrit H.F. Ellenberger, les experts ont été impressionnés par les cas de "meurtres immotivés 》, commis par des schizophrènes au cours de la phase initiale de la maladie. Ces meurtres constituaient souvent la première manifestation visible de la maladie » (p. 309).

\section{Prévention et traitement}

En d'autres termes il n'était pas toujours possible de la décéler 
et d'agir à titre préventif, avant la commission de l'acte. Or, il s'agissait de meurtres immotivés et imprévisibles;

... des catatoniques chroniques, ... pouvaient sortir brusquement de leur état de stupeur, se rendre dans la chambre d'un malade voisin, lui défoncer le crâne et retourner se replonger dans leur stupeur (p. 309).

On image aisément quel degré de rejet peut apparaître chez le profane face à une maladie constituant une menace aussi directe. Aussi H.F. Ellenberger s'empresse de souligner les aspects sociologiques de la schizophrénie "maladie de la civilisation" (p. 313) des «relations entre la schizophrénie et l'échelle sociale " et finalement le fait que «la schizophrénie... subit intensément l'effet du milieu culturel ambiant » (p. 317).

Ce chapitre permet aux non-spécialistes de pressentir la complexité socio-culturelle de certains phénomènes et constitue aussi une mise en garde destinée aux criminologues, comme à tous les intervenants.

"Certains malades, guéris spontanément", écrit l'auteur, «après une période de schizophrénie grave, sont passés au rang de bienfaiteurs de l'humanité », dans d'autres cas... "il faut mentionner... les écrits de certains schizophrènes " dont Gérard de Nerval, Holderlin ou Nelligan, entre autres, ou encore des œuvres des peintres dont le célèbre Adolf Wolffli.

En dernier lieu, H.F. Ellenberger souligne qu'au "point de vue scientifique, la schizophrénie a enrichi indirectement la médecine, la psychologie et la sociologie en stimulant la recherche sur un plan immense... tandis que sur le plan... philosophique, il n'est pas de maladie qui, autant que la schizophrénie (sauf peut-être l'épilepsie) ait permis de sonder les profondeurs de la nature humaine."

Un autre chapitre dans lequel H.F. Ellenberger traite expressément des aspects criminologiques, porte sur les délires chroniques. Au niveau de la définition: "Il s'agit de malades qui présentent une construction délirante permanente, qui engage toute leur personnalité, quoique à un degré moindre que dans les psychoses schizophréniques" (p. 319). "À l'intérieur de ce vaste cadre, on rencontre de nombreux cas difficiles à classer, mais aussi un certain nombre de types cliniques bien définis. La psychose hallucinatoire chronique, les délires de revendication et l'érotomanie, par exemple, sont des maladies aussi différenciées que le sont l'hébéphrénie ou la catatonie dans le cadre de la schizophrénie " (p. 319). 
Les aspects criminologiques de ces maladies sont examinés sous l'angle du danger de victimisation pour des tiers. L'auteur constate à cet égard que les délires d'imagination peuvent conduire aux escroqueries tandis que la psychose hallucinatoire chronique, ainsi que l'érotomanie, quand elle arrive au stade de la haine, peuvent conduire au meurtre.

"Les délirants chroniques les plus dangereux sont les paranoïaques revendicateurs et persécutés " (p. 330). Ils peuvent commettre un meurtre, ou encore des vols puisqu'il s'agit «dans leur esprit d'une reprise de biens dont on les a frustrés ").

On constate aisément, à partir de là, à quel point est tenue la limite entre le "malade" et le "délinquant ". Les plaidoieries, où les avocats de la défense développent la thèse que leur client (abandonné en bas âge, continuellement mal traité par la collectivité, a commis des actes répréhensibles à cause de toutes ces frustrations) n'est pas un délinquant, mais la victime innocente. ne manquent pas. Bien au contraire, les avocats puisent largement dans des définitions issues de la psychiatrie et les psychiatres ont souvent beaucoup de difficultés à établir un diagnostic dans le cadre d'une procédure judiciaire répressive par définition.

À l'opposé, se pose tout le problème de traitement "volontaire" ou obligatoire. En effet, comme le constate Henri Ellenberger, "l'Association de la psychothérapie et de la pharmacothérapie constitue le traitement recommandé pour ce type de patient 》 (p. 331).

Or, autant il est évident qu'un délinquant peut être porté à se faire passer pour un malade, si cela peut lui éviter une détention prolongée, autant il n'est pas du tout sûr qu'un emprisonnement ne sera pas préférable pour sa santé mentale que le traitement qui comprend l'absorption des médicaments pouvant avoir des divers effets secondaires.

En ce qui a trait à la psychothérapie, les spécialistes conviennent que le patient doit y participer, sous peine d'échec, ce qui signifie que la mesure ne doit pas être imposée, mais volontaire.

En pratique, on relève des malades qui ne veulent pas être traités, comme des délinquants qui préfèrent, il va sans dire, accepter n'importe quelle contrainte susceptible de raccourcir, ou à plus forte raison, d'éliminer une peine à purger. Le commentaire de Jean-Guy Fontaine sur les états limites (borderline), ajoute encore à la confusion toujours possible. "La pathologie borderline, écrit-il, a 
progressivement pris la place des névroses classiques, probablement à cause des changements sociaux et familiaux qui ont fait que les normes sont moins intransigeantes, que les lois sont plus aléatoires... » (p. 339, 340).

\section{Psychiatrie et évaluation des mineurs}

Comme on le constate en somme, les liens entre la science psychiatrique, les problèmes sociaux et la loi se tissent, à l'insu presque de ces diverses disciplines, à partir des expériences concrètes auxquelles les praticiens sont confrontés au jour le jour.

Un des mérites de l'ouvrage et non pas des moindres consiste dans la conception générale de son plan qui facilite l'accès des textes sur des problèmes particuliers sur lesquels le lecteur désire se documenter. Ȧ cet égard, nous ne retiendrons ici, en fonction de nos préoccupations, que les deux chapitres de la troisième partie qui portent spécifiquement sur les troubles psychiatriques selon les âges et qui concernent l'enfance d'âge préscolaire et scolaire, ainsi que l'adolescence.

On y trouvera sous la signature de Dr Suzanne Dongier et Dr Eva Lester certaines affirmations qui nous paraissent particulièrement liées à la pratique criminologique.

"Il est bien connu, écrivent ces deux auteurs, que la personnalité n'atteint son organisation définitive que vers la fin de l'adolescence : en conséquence, le diagnostic de trouble de la personnalité de l'enfant ne devrait être utilisé qu'à titre d'essai. "

Quelle magnifique mise en garde, pour tous ceux qui, conformément aux législations sur les jeunes délinquants contenues dans le code criminel, s'acharnent à faire examiner des enfants dont les comportements sont considérés comme délinquants et cela "dès l'âge de 7 ans, ou même avant "! On comprend à partir de là les confusions multiples qu'on retrace dans les dossiers judiciaires des mineurs, déclarés tantôt comme des "névrotiques", "psychotiques", ou autres et tantôt comme "normaux", au fil des examens effectués dès leur jeune âge, au nom de la prévention, bien que la signification réelle de ces examens est pratiquement nulle compte tenu de l'évolution de l'enfance. Ajoutons que, souvent, ces diagnostics, on ne peut plus douteux en fait, servent d'excuses aux praticiens et de moyen de stigmatisation aux autorités scolaires ou autres, en pénalisant ainsi encore davantage des mineurs qui ont surtout besoin de protection. 
Les deux auteurs constatent, par ailleurs, que :

Chez l'enfant, la pathologie de base de troubles de la personnalité est caractérisée par la tendance au passage à l'acte immédiat plutôt qu'à l'inhibition à retardement des pulsions sexuelles. Cette tendance provoque un comportement socialement inacceptable et crée des problèmes sérieux entre l'enfant et son environnement. En dépit de la nature souvent grave et de leurs actes antisociaux, ces enfants montrent peu d'anxiété ou de culpabilité et semblent incapables d'apprendre à partir de l'expérience (p. 409).

L'approche médicale, ou psychiatrique, ne facilite guère ici la compréhension réelle du phénomène. En effet, les enfants qui n'ont pas acquis une conscience suffisante des concepts tels que le bien ou le mal, les enfants insuffisamment socialisés par leur famille, peuvent tous "montrer peu d'anxiété ou de culpabilité " et ne pas savoir tirer, selon beaucoup de probabilité, aucun enseignement de leur expérience en dehors de l'apprentissage de la peur, ou de la haine de la justice des adultes.

En ce qui a trait au chapitre signé par Michel Lemay qui traite de l'adolescence, l'auteur constate :

Il est impossible d'étudier les troubles psychiques de l'adolescence sans les replacer dans le contexte de la situation vécue par l'adolescent au sein d'une société donnée... Outre le milieu familial, l'école et les groupes de pairs occupent une place privilégiée dans ses préoccupations et lui fournissent les moyens de valorisation dont il a absolument besoin (p. 419).

Face à cette affirmation, les relevés des dossiers judiciaires où on ne tient compte que de la situation familiale des enfants protégés, ou délinquants, puisque l'école ne fournit pas, ou se contente de peu de renseignements (généralement strictement liés avec les résultats relatifs aux notes obtenues et à la relation entre l'âge et le niveau du cycle scolaire), on se rend compte de l'énormité de certaines évaluations pourtant prétendument scientifiques.

En somme, tout en rendant les psychiatres responsables de plusieurs formes de stigmatisation des mineurs, ou encore en utilisant la science psychiatrique comme excuse pour éviter des interventions socio-éducatives, aussi indispensables puissent-elles être, on procède à une forme de distorsion de l'image que les praticiens peuvent avoir de l'enfant dont le dossier leur est confié.

\section{Le droit d'appel}

En terminant cette brève analyse de l'ensemble de l'ouvrage, 
mentionnons les deux derniers chapitres qui portent sur les législations concernant le malade mental, au Québec et en France. Faute de pouvoir les résumer, voici uniquement un extrait de la conclusion de Richard Michaud qui traite du Québec, relativement au droit d'appel.

... Nous ne croyons pas superflu, écrit-il, de répéter que toute restriction de liberté, toute suspension d'un droit et toute perte de privilèges légaux pour un malade ne devraient jamais être imposées, ni consenties par le législateur sans qu'elles ne s'accompagnent d'un recours devant un tribunal d'appel ou de révision, facilement accessible et investi des compétences et des pouvoirs nécessaires à son efficacité (p. 652).

L'auteur semble y reconnaître les difficultés du diagnostic de dangerosité, comme de tout autre, absolu et définitif. Il introduit implicitement le doute et la nécessité de tenir compte, non seulement de l'évolution des malades concernés mais aussi du vieux proverbe latin Errare humanum est soit du fait qu'un expert-psychiatrique et même plusieurs peuvent se tromper. Dans cette perspective, nous, qui sommes particulièrement intéressés par des problèmes des mineurs, ne pouvons nous empêcher de relever la mise en garde, qui ressort de ce précis magistral de la pratique de psychiatrie, en ce qui a trait à la stigmatisation des enfants et des adolescents.

Les termes scientifiques, mal compris par les praticiens et encore plus mal interprétés à l'occasion, sont exploités consciemment, ou automatiquement, tandis qu'aucun droit d'appel ne saurait compenser pour des dommages, ainsi occasionnés, puisqu'ils sont souvent irréversibles chez des êtres très jeunes.

En somme, autant le droit d'appel peut constituer une protection pour le malade mental adulte, autant au niveau des mineurs, il y a peut-être lieu de se demander si les évaluations psychiatriques ne devraient pas tout simplement être complètement éliminées des dossiers judiciaires, sociaux et scolaires.

Factuel et clair, le livre, dans son ensemble, explique plusieurs phénomènes et, en tant que tel, mérite, selon nous, de se trouver non seulement dans les bibliothèques des spécialistes, mais également chez tous ceux qui s'intéressent au dilemme fondamental des droits des malades mentaux qui sont toujours encore perçus par la société comme une menace. 